Hould selection of the most probable alternative improve if smailer size sequences of trials were used? This question was submitted to test by using different sizes of trial sequences. Sizes of sequences compared were: 5 , 8 , and 11. As before in sequence $=0$, the first 10 trials were considered as practice trials and used for computing initial proportions of reinforcements on each alternative for Trials 11-250. For sequence sizes of smaller sizes, however, proportions of reinforcements were computed for the size of the sequence. For example, with sequences $=5$, the proportions of reinforcements occurring for the five alternatives on Trial 15 were computed for Trials 11-15 and for Trial 16, for Trials 12-16. Similar types of analysis were computed for sequences of sizes 8 and 11 .

To determine if improvement in selecting the most probable alternative would occur when smaller sizes of sequences of trials were used, the following statistical analysis was made of these data. Frequency of most probable alternatives selected for blocks of 40 trials were counted for the six males and eight females as different groups for the three sequence sizes. In this way, a 2 by 3 by 6 analysis of variance for repeated measures was computed: two factors for sex, three factors for sequence sizes, and six factors for blocks of trials. Statistical significanes were obtained for sequence size, $\mathrm{F}(2,24)=4.63, \mathrm{p}<.05$, and for trials, $\mathrm{F}(5,60)=4.39, \quad \mathrm{p}<.01 ; \quad$ all other effects were nonsignificant. Examination of the means of the most probable responses for the three sequence sizes revealed that more responses were made in Sequence Size $8(\bar{x}=16.87)$ than in sequences of Sizes $11(\bar{x}=16.68)$ and $5(\bar{x}=15.88)$. Tests of the differences between means for the three sequences sizes by the Newman-Keuls procedure showed significant differences between sequences of Sizes 8 and 5 and Sequences 11 and 5 , but not between Sizes 11 and 8 . Learning to select the most probable alternative seems to occur with experience in the situation, and is related in some way to the size of the sequence of trials.

\section{Relationships Between Other Measures}

The relationships between three variables were also examined: the number of repetitions of response, $A_{i}$, on Trial $n+1$ if $\mathrm{II}_{\mathrm{i}}$ occurred on Trial $n$, the total number of most probable alternatives selected, and the total number of reinforcements received. Kendall's coefficients of correlation between repetition of responses on Trial $n+1$ if $\Pi_{i}$ occurred on Trial $n$ and the number of the most probable alternative selected was .67 , and between repetition of responses and number of most probable alternatives selected, .69. A $\tau=.74$ was obtained between the total number of most probable alternatives selected and the total number of reinforcements. All three coefficients were highly significant at $\mathrm{p}<.0005$.

These significant relations provide additional evidence in support of the previous analyses that learning to select the most probable alternative is related to the reinforcements obtained on previous selections of alternatives.

$$
\text { REFERENCES }
$$

BEACH, L, R, \& SHOENBERGER, R, W. Event salience and response frequency in a ten-alternative probability-learning situation. Journal of Experimental Psychology, 1965, 69, 312-316.

COTTON, J. W.. \& RECHTSCHAFFEN, A. Replication report: Two- and three-choice verbal conditioning phenomena. Journal of Experimental Psychology, 1958, 56, 96 .

ERICKSON, J. R. On learning several simultaneous probability-learning problems. Journal of Experimental Psychology, 1966. 72. 182-189.
ESTES, W. K. Probability learning. In A. W. Melton (Ed.), Categories of human learning. New York: Academic Press, 1964. Pp. 89-128.

GARDNER, R. A. Probability-learning with two and three choices. A merican Journal of Psychology, 1957, 70, 174-185.

GARDNER, R. A. Multiple-choice decision-behavior. American Journal of Psychology, 1958, 71, 710-717.

GARDNER, R. D.. \& FORSYTHE. J. B. Supplementary report: Two-choice decision behavior with many alternative events. Journal of Experimental Psychology, 1961, 62,631.

KOMORITA, S. S, Probability learning under equivalent data collection methods. Journal of Experimental Psychology, $1958,55,115-120$.

MCCORMACK, P. D. Spatial generalization and probability learning in a five-choice situation. American Journal of Psychology, 1959, 72, 135-138.

NEIMARK, E. D. Effects of type of nonreinforcement and number of alternative responses in two verbal conditioning situations. Journal of Experimental Psychology, 1956, 52, 209-219.

PAGE, E. B. Ordered hypotheses for multiple treatments: A significance test for linear ranks. Journal of American Statistical Association, 1963, 58, 216-230.

\title{
The effects of irrelevant dimensions and stimuli on two-choice discrimination learning by children
}

\author{
LESTER M. HYMAN \\ Michigan State University, East Lansing, Mich. 48823
}

Eight 9-year-old children were presented 24 eight-trial simultaneous two-choice visual discrimination problems. Each problem contained eight different stimuli. In half of the problems the stimuli varied along two dimensions with one dimension irrelevant, and for the other 12 problems the stimuli varied along three dimensions with two dimensions irrelevant. Even though the number of irrelevant stimuli was the same, the two-irrelevant-dimension problems were significantly more difficult than the one-irrelevant-dimension problems. There was significant learning on both types of problems and no significant interactions.

Several recent theories of discrimination learning (Zeaman \& House, 1963; Lovejoy, 1965; Restle, 1955) predict differences in the difficulty of acquisition of a discrimination as a function of the number of redundant relevant cues or dimensions. A number of studies have clearly shown enhancement in acquisition, which is directly related to increasing numbers of redundant relevant cues and dimensions (Hyman, 1967; Osler \& Kofsky, 1965). The same theories also predict that an increase in the number of irrelevant dimensions or cues should lead to an increase in the difficulty of acquisition. Altough this is generally true (Zeaman \& House, 1963), in most experimental demonstrations of the effects of irrelevant dimensions, the number of dimensions and the number of different cues, or stimuli, presented to the Ss has been correlated (Tighe \& Tighe, 1969). It is, therefore, not clear whether dimensional selectivity or cue selectivity is the major source of variability in speed of acquisition when the number of irrelevant dimensions is varied.

The present study allowed for varying the number of irrelevant dimensions while keeping the number of different cues presented constant by varying the number of cues presented along the irrelevant dimensions. It was hypothesized that the number of irrelevant dimensions and not the total number of irrelevant cues would have the greatest effect on the difficulty of two-choice visual discrimination problems.

\section{SUBJECTS}

Four boys and four girls, ranging in age from 9 years 11 months to 10 years 11 months, served as Ss. The children were all residents of married student housing at Michigan State University. None of the children had previously served in similar experiments. 


\section{STIMULI}

The two-dimensional stimuli (one irrelevant dimension-1 ID) consisted of $90 \quad 2^{1 / 2} \times 2^{1 / 2}$ in, white cards containing colored drawings of 10 simple shapes (bell, cross, star, pentagon, diamond, circle, square, triangle, heart, and $\mathrm{T}$ ) colored by pencil in nine shades (gray, yellow, red, blue, dark green, black, orange, brown, and purple). The three-dimensional stimulus (two irrelevant dimensions) consisted of $2^{1 / 2} \times 2^{1 / 2}$ in. white cards containing all possible combinations of seven figures (heart, triangle, square, circle, diamond, star, and T), 10 colors (gray, yellow, red, blue, dark green, light green, black, orange, brown, and purple), and four textured backgrounds (dense vertical lines, dense oblique lines, sparse vertical lines, and sparse oblique lines). The large numbers of forms and colors used with the three-dimensional stimuli allowed two and three-dimensional problems to be sequenced with no repetition of particular cues on adjacent problems. DESIGN

Each $S$ received eight trials on 12 two-dimensional (1 ID) and 12 three-dimensional (2 ID) two-choice simultaneous discrimination problems. The 24 problems were presented in two daily sessions lasting approximately $1 / 2 \mathrm{~h}$ each. Half of each type of problem were presented in Session 1 and half in Session 2. Balancing among the different types of problems, each $S$ received six 1-ID problems each session, three with form relevant and three with color relevant. Each $S$ also received six 2-ID problems each session, two with form relevant, two with color relevant, and two with texture relevant. Each 2-ID problem contained eight different stimuli, i.e. all combinations of two cues of three dimensions. Each 1-ID problem also contained eight different cues, the combinations of the two cues from the relevant dimension with four different cues of the irrelevant dimension. Each stimulus card appeared twice in a given problem, and a given positive and negative stimulus card were only paired once in a problem.

\section{PROCEDURE}

Prior to the first trial, the $\mathbf{S}$ was informed that he would be shown pairs of cards where one of each pair was "correct" and the other one was "wrong." He was instructed to pick the member of each pair he thought was "correct." The $S$ was also informed that he would receive a reward at the end of the session if he was able to do "well" at guessing the correct cards. No other information was given the $\mathrm{S}$.

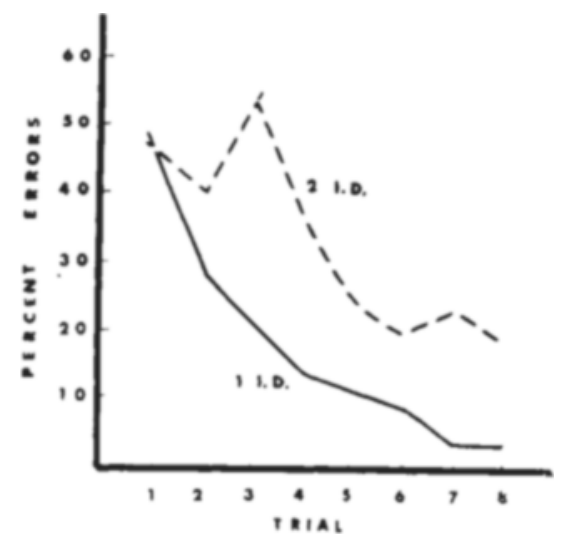

Figure 1

\section{PRETRAINING}

Each $S$ was given one 1-ID and one 2-ID problem for a maximum of 25 trials. Criterion for entry to the experiment was eight successive correct responses on both problems. One $S$ who failed pretraining was dropped from the experiment.

\section{APPARATUS}

The apparatus used was a modified Wisconsin General'Test Apparatus. It consisted of a table with a partition containing a one-way mirror separating $E$ from $S$ and a 30 -in.-wide drawer that could be pulled back out of sight of the $S$ for rearranging the stimuli. The stimuli were placed approximately $15 \mathrm{in}$. apart on the drawer. To initiate a trial, the E slid the drawer containing the two stimulus cards in front of the $S$. When the $S$ picked up one of the two cards, he was informed if his guess was "correct" or "wrong." The drawer and stimuli were then pulled back out of sight of the $\mathbf{S}$ for arranging stimuli for the next trial.

\section{RESULTS}

The percentage of errors per condition as a function of trials is presented in Fig. 1. A preliminary analysis of variance showed no significant differences among relevant dimensions within condition. After collapsing the relevant dimension

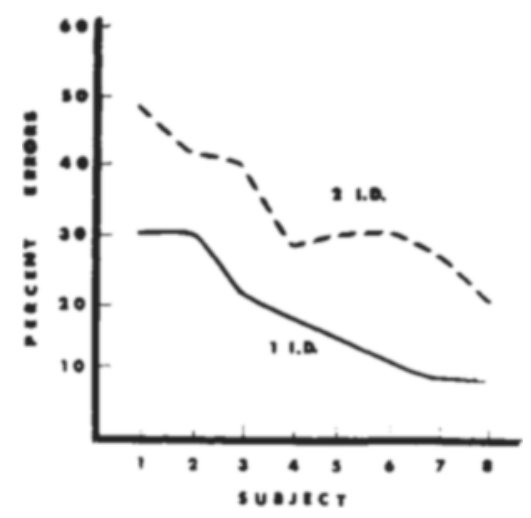

Figure 2 variable, a threc-way analusis of variance ( 2 by 7 by 8 ) was computed on conditions (one or two irrelevant dimensions), trials (Trials $2-8$ ), and Ss. None of the two-way interactions was significant, although Conditions by Trials approached significance $(F=2.02, \quad \mathrm{df}=6 / 42, \mathrm{p}-.05)$. Both testable meain effects were highly significant (conditions, $F=182.3$, $\mathrm{df}=1 / 7, \quad \mathrm{p}<.001 ;$ trials,$\quad F=15.3$, df $=6 / 42, \quad p<.001)$. Although the significance of the third main variable, $\mathrm{Ss}$, is not testable, it was clear that there were large differences among Ss. Figure 2 presents the percentage of errors per condition for each $\mathrm{S}$. The Ss were ordered by their performance on the 1-ID condition. Additional analysis of the data showed no differences as a function of sex and no effect of sessions.

\section{DISCUSSION}

The results of this experiment clearly demonstrate that the number of irrelevant dimensions, rather than the number of different cues presented, is the important variable in determining difficulty of a discrimination problem with one relevant binary dimension. This finding is clearly more supportive of attention theories and hypothesis testing theories than of either cue selection notions or sampling theories, such as stimulus sampling theory. It would seem that, by 9 years of age, children do respond primarily to dimensions or abstract attributes rather than to concrete cues. It is important to note that all the children in the experiment showed the effect. It is also noteworthy that all the Ss showed significant learning for problems that contained one irrelevant dimension, while three of them did not demonstrate any improvement over chance in the two-irrelevantdimension problems.

\section{REFERENCES}

HYMAN, L. M. The effect of redundant patterns on retardate discrimination learning. Psychonomic Science, 1967, 9, 195-196.

LOVEJOY. E. An attention theory of discrimination learning. Journal of Mathematical Psychology, 1965, 2 . 342-362.

OSLER, S. F., \& KOFSKY, E. Stimulus uncertainty as a variable in the development of conceptual ability. Journal of Experimental Child Psychology, 1965, 2, 264-279.

RESTLE, F. A theory of discrimination learning. Psychological Review, 1955,62, $11-20$

TIGHE, L. S., \& TIGHE, T. J. Transfer from perceptual pretraining as a function of number of task dimensions. Journal of Experimental Child Psychology, 1969, 8 . 494-502.

ZEAMAN, D., \& HOUSE, B. J. An attention theory of retardate discrimination learning. In N. R. Ellis (Ed.), Handbook of mental deficiency: Psychological theory and research. New York: McGraw-Hill, 1963. Pp. 159-223. 